\title{
Analysis and behavior of high-strength rectangular CFT columns
}

\author{
Z. Lai ${ }^{a *}$ and A. H. Varmab \\ ${ }^{a}$ Department of Civil Engineering, Fuzhou University, China \\ byles School of Civil Engineering, Purdue University, USA \\ *corresponding author, e-mail address: laizhichao@gmail.com
}

\begin{abstract}
The current AISC Specification (AISC 360-16) specifies the material strength limits for concrete-filled steel tube (CFT) columns. According to AISC 360-16, the steel yield stress $\left(F_{y}\right)$ for CFT columns should not exceed $525 \mathrm{MPa}$, and the concrete compressive strength $\left(f^{\prime}{ }_{c}\right)$ should not exceed $70 \mathrm{MPa}$. CFT columns are classified as high strength if either $F_{y}$ or $f_{c}^{\prime}$ exceeds these specified limits, and are classified as conventional strength if both $F_{y}$ and $f_{c}^{\prime}$ are less than or equal to the limits. Due to lack of adequate research and comprehensive design equations, AISC 360-16 does not endorse the use of high-strength materials for CFT columns. This paper makes a contribution towards addressing this gap using a two-step approach. The first step consists of compiling an experimental database of high-strength rectangular CFT column tests in the literature and evaluating the possibility of extending the current AISC 360-16 design equations to high-strength rectangular CFT columns. The second step consists of developing and benchmarking detailed 3D nonlinear finite element models for predicting the behavior of high-strength CFT columns from the database. The benchmarked models are being used to perform comprehensive parametric studies to address gaps in the database and propose design equations for high-strength rectangular CFT members, which will be part of a future paper.
\end{abstract}

Keywords: High-strength; CFT columns; experimental database; finite element analysis; design.

\section{Introduction}

Concrete-filled steel tube (CFT) members are usually comprised of rectangular or circular steel tubes filled with concrete. CFT members can be more efficient than reinforced concrete or structural steel members due to the synergistic interaction between the steel tube and concrete infill. The steel tube provides confinement to the concrete infill, while the concrete infill delays the local buckling of the steel tube [1] [2][3]. As an innovative structural component, CFT members have been used widely around the world in various structures. For example, they have been used as: (i) chords in composite arch bridges [4], (ii) mega columns in high-rise buildings [5], (iii) piles in floodwall structures [6], and (iv) bridge piers [7].

The current AISC Specification (AISC 36016) [8] does not endorse the use of high-strength materials for CFT columns. According to AISC 360-16 [8], the steel yield stress $\left(F_{y}\right)$ should not exceed $525 \mathrm{MPa}$, and the concrete compressive strength $\left(f^{\prime} c\right)$ should not exceed $70 \mathrm{MPa}$. CFT members are classified as high strength if either $F_{y}$ or $f^{\prime}{ }_{c}$ exceeds these specified limits. CFT members are classified as conventional strength if both $F_{y}$ and $f^{\prime}{ }_{c}$ are less than or equal to the limits.

Experimental studies have been conducted to evaluate the behavior of conventional-strength CFT members as columns, beams, and beamcolumns. These studies have been summarized independently by several researchers in [1] [9] [10] [11] [12]. These experimental tests indicate that the behavior and strength of conventionalstrength CFT members depend on parameters such as the concrete compressive strength $\left(f^{\prime} c\right)$, steel yield stress $\left(F_{y}\right)$, tube width-to-thickness ratio $(b / t)$ or diameter-to-thickness ratio $(D / t)$, and member length $(L)$.

Based on the findings from these prior investigations, AISC 360-16 [8] provides design 
provisions for conventional-strength CFT members, which include: (i) steel tube slenderness limits (i.e., tube width-to-thickness limits) to categorize CFT members into compact, noncompact, and slender; and (ii) design equations for estimating the compressive, flexural and beam-column strength of CFT members. The authors have presented the basis (development and verification) of these design provisions elsewhere in [1] [3] [12].

Several researchers have experimentally investigated the behavior of high-strength rectangular CFT columns, including Cederwall et al. [13], Varma [14], Uy [15], Liu et al. [16], Mursi and Uy [17], Sakino et al. [18], Liu [19], Lue et al. [20], Aslani et al. [21], Xiong et al. [22], and Khan et al. [23] among others. These prior studies have provided valuable insights into the fundamental behavior of high-strength rectangular CFT columns. However, there is a lack of comprehensive design equations based on the compilation of these studies. Consequently, AISC 360-16 [8] does not endorse the use of high-strength rectangular CFT columns.

This paper makes a contribution towards addressing this gap using a two-step approach. The first step consists of compiling an experimental database of high-strength rectangular CFT column tests in the literature and evaluating the possibility of extending the current AISC 360-16 design equations to highstrength rectangular CFT columns. The second step consists of developing and benchmarking detailed 3D nonlinear finite element models for predicting the behavior of high-strength CFT columns from the database. The benchmarked models are being used to perform comprehensive parametric studies to address gaps in the database and propose design equations for high-strength rectangular CFT members, which will be part of a future paper.

\section{Experimental database}

As discussed in the previous section, several experimental tests have been conducted to evaluate the behavior and strength of highstrength rectangular CFT columns. This section compiles an experimental database by reviewing these tests. A total of 130 tests on high-strength CFT columns were included in the database. These specimens were categorized into three types depending on the material strengths of the steel tube and concrete infill, as identified in Table 1. The first type (HS-CC) consisted of 40 CFT columns with high-strength steel tube and conventional-strength concrete infill. The second type (CS-HC) consisted of 41 specimens with conventional-strength steel tube and highstrength concrete infill. The third type (HS-HC) consisted of 49 specimens with high-strength steel tube and concrete infill. Table 1 summarizes the details of the 109 short column specimens with length-to-depth ratio $(L / H)$ less than or equal to 6.0 , while Table 2 summarizes the details of the 21 slender column specimens with $L / H$ greater than 6.0.

Fig. 1(a) and Fig. 1(b) compares the strengths calculated using the AISC design equation $\left(P_{n}\right)$ with those obtained from the experiments $\left(P_{\text {exp }}\right)$ for the short and slender column specimens, respectively. Fig. 1(a) indicates that the AISC design equations can reasonably estimate the strength of high-strength rectangular CFT short columns. The mean $P_{\text {exp }} / P_{n}$ ratio is 1.07 , and the corresponding coefficient of variation is 0.09 . These comparisons also indicate that the AISC design equations are conservative for columns with compact sections and slightly unconservative for columns with noncompact sections. Due to lack of test data, no conclusions can be made regarding the conservatism of the AISC 360-16 equations for designing (i) highstrength rectangular CFT short columns with slender sections or (ii) high-strength rectangular CFT slender columns. Additional data points are required. This was addressed by conducting finite element analysis using the FEM models developed and benchmarked in the next section.

\section{Finite element models}

This section develops and benchmarks detailed 3D nonlinear FEM models for highstrength rectangular CFT columns. The FEM models were developed using ABAQUS [24]. Details of the FEM models include: (i) element types, (ii) contact interaction, (iii) steel and concrete material models, (iv) geometric imperfections, (v) boundary conditions, and (vi) analysis method. Most of these details are similar to those presented by the authors previously [1] for conventional-strength 
Table 1. High-strength rectangular CFT column tests: short columns $(L / H \leq 6.0)$.

\begin{tabular}{|c|c|c|c|c|c|c|c|c|c|c|c|c|c|c|c|}
\hline Reference & $\begin{array}{c}\text { Specimen } \\
\text { Type }\end{array}$ & Specimen ID & $\begin{array}{c}L \\
(\mathrm{~mm})\end{array}$ & $\begin{array}{c}B \\
(\mathrm{~mm})\end{array}$ & $\begin{array}{c}t_{f} \\
(\mathrm{~mm})\end{array}$ & $b / t_{f}$ & $\begin{array}{c}H \\
(\mathrm{~mm})\end{array}$ & $\begin{array}{c}t_{w} \\
(\mathrm{~mm})\end{array}$ & $h / t_{w}$ & $\lambda_{\text {coeff }}$ & $L / H$ & $\begin{array}{c}F_{y} \\
(\mathrm{MPa})\end{array}$ & $\begin{array}{c}f^{\prime}{ }_{c} \\
(\mathrm{MPa})\end{array}$ & $\begin{array}{c}E_{c} \\
(\mathrm{GPa})\end{array}$ & $\begin{array}{l}P_{\exp } \\
(\mathrm{kN})\end{array}$ \\
\hline \multirow{6}{*}{$\begin{array}{c}\text { Cederwall } \\
\text { et al. } \\
(1990)\end{array}$} & CS-HC & $3-1$ & 500.0 & 120.0 & 8.00 & 13.0 & 120.0 & 8.00 & 13.0 & 0.53 & 4.2 & 327.0 & 96.0 & 46.37 & 2040.0 \\
\hline & CS-HC & $4-1$ & 500.0 & 120.0 & 8.00 & 13.0 & 120.0 & 8.00 & 13.0 & 0.61 & 4.2 & 439.0 & 96.0 & 46.37 & 2240.0 \\
\hline & CS-HC & $8-1$ & 500.0 & 120.0 & 8.00 & 13.0 & 120.0 & 8.00 & 13.0 & 0.52 & 4.2 & 323.0 & 103.0 & 48.03 & 2270.0 \\
\hline & CS-HC & $9-1$ & 500.0 & 120.0 & 8.00 & 13.0 & 120.0 & 8.00 & 13.0 & 0.57 & 4.2 & 379.0 & 103.0 & 48.03 & 2680.0 \\
\hline & CS-HC & $13-1$ & 500.0 & 120.0 & 8.00 & 13.0 & 120.0 & 8.00 & 13.0 & 0.57 & 4.2 & 390.0 & 80.0 & 42.33 & 2300.0 \\
\hline & CS-HC & $14-1$ & 500.0 & 120.0 & 8.00 & 13.0 & 120.0 & 8.00 & 13.0 & 0.57 & 4.2 & 379.0 & 80.0 & 42.33 & 2290.0 \\
\hline \multirow{4}{*}{$\begin{array}{l}\text { Varma } \\
(2000)\end{array}$} & HS-HC & SC-32-80 & 1200.0 & 305.0 & 8.90 & 32.3 & 305.0 & 8.90 & 32.3 & 1.71 & 3.9 & 560.0 & 110.0 & 49.64 & 14116.0 \\
\hline & HS-HC & SC- $48-80$ & 1200.0 & 305.0 & 6.10 & 48.0 & 305.0 & 6.10 & 48.0 & 2.76 & 3.9 & 660.0 & 110.0 & 49.64 & 12307.0 \\
\hline & CS-HC & SC-32-46 & 1200.0 & 305.0 & 8.60 & 33.5 & 305.0 & 8.60 & 33.5 & 1.20 & 3.9 & 259.0 & 110.0 & 49.64 & 11390.0 \\
\hline & CS-HC & SC-48-46 & 1200.0 & 305.0 & 5.80 & 50.6 & 305.0 & 5.80 & 50.6 & 2.45 & 3.9 & 471.0 & 110.0 & 49.64 & 11568.0 \\
\hline \multirow{6}{*}{ Uy (2001) } & HS-CC & HSS1 & 330.0 & 110.0 & 5.00 & 20.0 & 110.0 & 5.00 & 20.0 & 1.22 & 3.0 & 750.0 & 28.0 & 25.04 & 1836.0 \\
\hline & HS-CC & HSS2 & 330.0 & 110.0 & 5.00 & 20.0 & 110.0 & 5.00 & 20.0 & 1.22 & 3.0 & 750.0 & 28.0 & 25.04 & 1832.0 \\
\hline & HS-CC & HSS8 & 480.0 & 160.0 & 5.00 & 30.0 & 160.0 & 5.00 & 30.0 & 1.84 & 3.0 & 750.0 & 30.0 & 25.92 & 2868.0 \\
\hline & HS-CC & HSS9 & 480.0 & 160.0 & 5.00 & 30.0 & 160.0 & 5.00 & 30.0 & 1.84 & 3.0 & 750.0 & 30.0 & 25.92 & 2922.0 \\
\hline & HS-CC & HSS14 & 630.0 & 210.0 & 5.00 & 40.0 & 210.0 & 5.00 & 40.0 & 2.45 & 3.0 & 750.0 & 30.0 & 25.92 & 3710.0 \\
\hline & HS-CC & HSS15 & 630.0 & 210.0 & 5.00 & 40.0 & 210.0 & 5.00 & 40.0 & 2.45 & 3.0 & 750.0 & 30.0 & 25.92 & 3483.0 \\
\hline \multirow{19}{*}{$\begin{array}{l}\text { Liu et al. } \\
\text { (2003) }\end{array}$} & HS-HC & C1-1 & 300.0 & 100.3 & 4.18 & 22.0 & 98.2 & 4.18 & 21.5 & 1.15 & 3.1 & 550.0 & 70.8 & 39.83 & 1490.0 \\
\hline & HS-HC & $\mathrm{C} 1-2$ & 300.0 & 101.5 & 4.18 & 22.3 & 100.6 & 4.18 & 22.1 & 1.17 & 3.0 & 550.0 & 70.8 & 39.83 & 1535.0 \\
\hline & HS-HC & $\mathrm{C} 2-1$ & 300.0 & 101.2 & 4.18 & 22.2 & 101.1 & 4.18 & 22.2 & 1.16 & 3.0 & 550.0 & 82.1 & 42.89 & 1740.0 \\
\hline & HS-HC & $\mathrm{C} 2-2$ & 300.0 & 100.7 & 4.18 & 22.1 & 100.4 & 4.18 & 22.0 & 1.16 & 3.0 & 550.0 & 82.1 & 42.89 & 1775.0 \\
\hline & HS-HC & $\mathrm{C} 3$ & 540.0 & 182.8 & 4.18 & 41.7 & 181.2 & 4.18 & 41.3 & 2.19 & 3.0 & 550.0 & 70.8 & 39.83 & 3590.0 \\
\hline & HS-HC & $\mathrm{C} 4$ & 540.0 & 181.8 & 4.18 & 41.5 & 180.4 & 4.18 & 41.2 & 2.18 & 3.0 & 550.0 & 82.1 & 42.89 & 4210.0 \\
\hline & HS-HC & C5-1 & 360.0 & 120.7 & 4.18 & 26.9 & 80.1 & 4.18 & 17.2 & 1.41 & 4.5 & 550.0 & 70.8 & 39.83 & 1450.0 \\
\hline & HS-HC & C5-2 & 360.0 & 119.3 & 4.18 & 26.5 & 80.6 & 4.18 & 17.3 & 1.39 & 4.5 & 550.0 & 70.8 & 39.83 & 1425.0 \\
\hline & HS-HC & C6-1 & 360.0 & 119.6 & 4.18 & 26.6 & 80.6 & 4.18 & 17.3 & 1.40 & 4.5 & 550.0 & 82.1 & 42.89 & 1560.0 \\
\hline & HS-HC & C6-2 & 360.0 & 120.5 & 4.18 & 26.8 & 80.6 & 4.18 & 17.3 & 1.41 & 4.5 & 550.0 & 82.1 & 42.89 & 1700.0 \\
\hline & HS-HC & C7-1 & 540.0 & 179.7 & 4.18 & 41.0 & 121.5 & 4.18 & 27.1 & 2.15 & 4.4 & 550.0 & 70.8 & 39.83 & 2530.0 \\
\hline & HS-HC & C8-1 & 540.0 & 180.4 & 4.18 & 41.2 & 119.8 & 4.18 & 26.7 & 2.16 & 4.5 & 550.0 & 82.1 & 42.89 & 2970.0 \\
\hline & HS-HC & C8-2 & 540.0 & 179.2 & 4.18 & 40.9 & 121.3 & 4.18 & 27.0 & 2.14 & 4.5 & 550.0 & 82.1 & 42.89 & 2590.0 \\
\hline & HS-HC & C9-1 & 480.0 & 160.2 & 4.18 & 36.3 & 81.4 & 4.18 & 17.5 & 1.90 & 5.9 & 550.0 & 70.8 & 39.83 & 1710.0 \\
\hline & HS-HC & C9-2 & 480.0 & 160.7 & 4.18 & 36.4 & 80.5 & 4.18 & 17.3 & 1.91 & 6.0 & 550.0 & 70.8 & 39.83 & 1820.0 \\
\hline & HS-HC & C10-1 & 480.0 & 160.1 & 4.18 & 36.3 & 81.0 & 4.18 & 17.4 & 1.90 & 5.9 & 550.0 & 82.1 & 42.89 & 1880.0 \\
\hline & HS-HC & $\mathrm{C} 10-2$ & 480.0 & 160.6 & 4.18 & 36.4 & 80.1 & 4.18 & 17.2 & 1.91 & 6.0 & 550.0 & 82.1 & 42.89 & 2100.0 \\
\hline & HS-HC & C11-1 & 600.0 & 199 & 4.18 & 45.6 & 101.2 & 4.18 & 22.2 & 2.39 & 5.9 & 550.0 & 70.8 & 39.83 & 2350.0 \\
\hline & HS-HC & C12-1 & 600.0 & 199.2 & 4.18 & 45.7 & 102.1 & 4.18 & 22.4 & 2.39 & 5.9 & 550.0 & 82.1 & 42.89 & 2900.0 \\
\hline \multirow{4}{*}{$\begin{array}{l}\text { Mursi and } \\
\text { Uy (2004) }\end{array}$} & HS-CC & SH-C110 & 430.0 & 120.0 & 5.00 & 22.0 & 120.0 & 5.00 & 22.0 & 1.36 & 3.6 & 761.0 & 20.0 & 21.17 & 1835.0 \\
\hline & HS-CC & SH-C160 & 580.0 & 170.0 & 5.00 & 32.0 & 170.0 & 5.00 & 32.0 & 1.97 & 3.4 & 761.0 & 20.0 & 21.17 & 2831.0 \\
\hline & HS-CC & SH-C210 & 730.0 & 220.0 & 5.00 & 42.0 & 220.0 & 5.00 & 42.0 & 2.59 & 3.3 & 761.0 & 20.0 & 21.17 & 3609.0 \\
\hline & HS-CC & SH-C260 & 880.0 & 270.0 & 5.00 & 52.0 & 270.0 & 5.00 & 52.0 & 3.21 & 3.3 & 761.0 & 20.0 & 21.17 & 3950.0 \\
\hline \multirow[t]{25}{*}{ Sakino et } & CS-HC & CR4-A-8 & 444.0 & 148.0 & 4.38 & 31.8 & 148.0 & 4.38 & 31.8 & 1.15 & 3.0 & 262.0 & 77.0 & 41.53 & 2108.0 \\
\hline & CS-HC & CR4-C-8 & 645.0 & 215.0 & 4.38 & 47.1 & 215.0 & 4.38 & 47.1 & 1.70 & 3.0 & 262.0 & 80.3 & 42.41 & 3837.0 \\
\hline & CS-HC & CR4-D-8 & 972.0 & 324.0 & 4.38 & 72.0 & 324.0 & 4.38 & 72.0 & 2.60 & 3.0 & 262.0 & 80.3 & 42.41 & 7481.0 \\
\hline & HS-CC & CR6-A-2 & 432.0 & 144.0 & 6.36 & 20.6 & 144.0 & 6.36 & 20.6 & 1.15 & 3.0 & 618.0 & 25.4 & 23.85 & 2572.0 \\
\hline & HS-CC & CR6-A-4-1 & 432.0 & 144.0 & 6.36 & 20.6 & 144.0 & 6.36 & 20.6 & 1.15 & 3.0 & 618.0 & 40.5 & 30.12 & 2808.0 \\
\hline & HS-CC & CR6-A-4-2 & 432.0 & 144.0 & 6.36 & 20.6 & 144.0 & 6.36 & 20.6 & 1.15 & 3.0 & 618.0 & 40.5 & 30.12 & 2765.0 \\
\hline & HS-HC & CR6-A-8 & 432.0 & 144.0 & 6.36 & 20.6 & 144.0 & 6.36 & 20.6 & 1.15 & 3.0 & 618.0 & 77.0 & 41.53 & 3399.0 \\
\hline & HS-CC & CR6-C-2 & 633.0 & 211.0 & 6.36 & 31.2 & 211.0 & 6.36 & 31.2 & 1.73 & 3.0 & 618.0 & 25.4 & 23.85 & 3920.0 \\
\hline & HS-CC & CR6-C-4-1 & 633.0 & 211.0 & 6.36 & 31.2 & 211.0 & 6.36 & 31.2 & 1.73 & 3.0 & 618.0 & 40.5 & 30.12 & 4428.0 \\
\hline & HS-CC & CR6-C-4-2 & 633.0 & 211.0 & 6.36 & 31.2 & 211.0 & 6.36 & 31.2 & 1.73 & 3.0 & 618.0 & 40.5 & 30.12 & 4484.0 \\
\hline & HS-HC & CR6-C-8 & 633.0 & 211.0 & 6.36 & 31.2 & 211.0 & 6.36 & 31.2 & 1.73 & 3.0 & 618.0 & 77.0 & 41.53 & 5758.0 \\
\hline & HS-CC & CR6-D-2 & 957.0 & 319.0 & 6.36 & 48.2 & 319.0 & 6.36 & 48.2 & 2.68 & 3.0 & 618.0 & 25.4 & 23.85 & 6320.0 \\
\hline & HS-CC & CR6-D-4-1 & 957.0 & 319.0 & 6.36 & 48.2 & 319.0 & 6.36 & 48.2 & 2.68 & 3.0 & 618.0 & 41.1 & 30.34 & 7780.0 \\
\hline & HS-CC & CR6-D-4-2 & 954.0 & 318.0 & 6.36 & 48.0 & 318.0 & 6.36 & 48.0 & 2.67 & 3.0 & 618.0 & 41.1 & 30.34 & 7473.0 \\
\hline & HS-HC & CR6-D-8 & 957.0 & 319.0 & 6.36 & 48.2 & 319.0 & 6.36 & 48.2 & 2.68 & 3.0 & 618.0 & 85.1 & 43.66 & 10357.0 \\
\hline & HS-CC & CR8-A-2 & 360.0 & 120.0 & 6.47 & 16.5 & 120.0 & 6.47 & 16.5 & 1.07 & 3.0 & 835.0 & 25.4 & 23.85 & 2819.0 \\
\hline & HS-CC & CR8-A-4-1 & 360.0 & 120.0 & 6.47 & 16.5 & 120.0 & 6.47 & 16.5 & 1.07 & 3.0 & 835.0 & 40.5 & 30.12 & 2957.0 \\
\hline & HS-CC & CR8-A-4-2 & 360.0 & 120.0 & 6.47 & 16.5 & 120.0 & 6.47 & 16.5 & 1.07 & 3.0 & 835.0 & 40.5 & 30.12 & 2961.0 \\
\hline & HS-HC & CR8-A-8 & 357.0 & 119.0 & 6.47 & 16.4 & 119.0 & 6.47 & 16.4 & 1.06 & 3.0 & 835.0 & 77.0 & 41.53 & 3318.0 \\
\hline & HS-CC & CR8-C-2 & 525.0 & 175.0 & 6.47 & 25.0 & 175.0 & 6.47 & 25.0 & 1.62 & 3.0 & 835.0 & 25.4 & 23.85 & 4210.0 \\
\hline & HS-CC & CR8-C-4-1 & 525.0 & 175.0 & 6.47 & 25.0 & 175.0 & 6.47 & 25.0 & 1.62 & 3.0 & 835.0 & 40.5 & 30.12 & 4493.0 \\
\hline & HS-CC & CR8-C-4-2 & 525.0 & 175.0 & 6.47 & 25.0 & 175.0 & 6.47 & 25.0 & 1.62 & 3.0 & 835.0 & 40.5 & 30.12 & 4542.0 \\
\hline & HS-HC & CR8-C-8 & 525.0 & 175.0 & 6.47 & 25.0 & 175.0 & 6.47 & 25.0 & 1.62 & 3.0 & 835.0 & 77.0 & 41.53 & 5366.0 \\
\hline & HS-CC & CR8-D-2 & 795.0 & 265.0 & 6.47 & 39.0 & 265.0 & 6.47 & 39.0 & 2.52 & 3.0 & 835.0 & 25.4 & 23.85 & 6546.0 \\
\hline & HS-CC & CR8-D-4-1 & 792.0 & 264.0 & 6.47 & 38.8 & 264.0 & 6.47 & 38.8 & 2.51 & 3.0 & 835.0 & 41.1 & 30.34 & 7117.0 \\
\hline
\end{tabular}


Table 1 (continued)

\begin{tabular}{|c|c|c|c|c|c|c|c|c|c|c|c|c|c|c|c|}
\hline Reference & $\begin{array}{c}\text { Specimen } \\
\text { Type }\end{array}$ & Specimen ID & $\begin{array}{c}L \\
(\mathrm{~mm})\end{array}$ & $\begin{array}{c}B \\
(\mathrm{~mm})\end{array}$ & $\begin{array}{c}t_{f} \\
(\mathrm{~mm})\end{array}$ & $b / t_{f}$ & $\begin{array}{c}H \\
(\mathrm{~mm})\end{array}$ & $\begin{array}{c}t_{w} \\
(\mathrm{~mm})\end{array}$ & $h / t_{w}$ & $\lambda_{\text {coeff }}$ & $L / H$ & $\begin{array}{c}F_{y} \\
(\mathrm{MPa})\end{array}$ & $\begin{array}{c}f^{\prime}{ }_{c} \\
(\mathrm{MPa})\end{array}$ & $\begin{array}{c}E_{c} \\
(\mathrm{GPa})\end{array}$ & $\begin{array}{l}P_{\exp } \\
(\mathrm{kN})\end{array}$ \\
\hline \multirow{12}{*}{$\begin{array}{l}\text { Sakino et } \\
\text { al. (2004) }\end{array}$} & $\mathrm{HS}-\mathrm{CC}$ & CR8-D-4-2 & 795.0 & 265.0 & 6.47 & 39.0 & 265.0 & 6.47 & 39.0 & 2.52 & 3.0 & 835.0 & 41.1 & 30.34 & 7172.0 \\
\hline & HS-HC & CR8-D-8 & 795.0 & 265.0 & 6.47 & 39.0 & 265.0 & 6.47 & 39.0 & 2.52 & 3.0 & 835.0 & 80.3 & 42.41 & 8990.0 \\
\hline & $\mathrm{CS}-\mathrm{HC}$ & CR4-A-9 & 633.0 & 211.0 & 5.48 & 36.5 & 211.0 & 5.48 & 36.5 & 1.40 & 3.0 & 294.0 & 91.1 & 45.18 & 4773.0 \\
\hline & $\mathrm{CS}-\mathrm{HC}$ & CR4-C-9 & 633.0 & 211.0 & 4.50 & 44.9 & 211.0 & 4.50 & 44.9 & 1.67 & 3.0 & 277.0 & 91.1 & 45.18 & 4371.0 \\
\hline & HS-CC & CR6-A-4-3 & 633.0 & 211.0 & 8.83 & 21.9 & 211.0 & 8.83 & 21.9 & 1.13 & 3.0 & 536.0 & 39.1 & 29.60 & 5898.0 \\
\hline & HS-HC & CR6-A-9 & 633.0 & 211.0 & 8.83 & 21.9 & 211.0 & 8.83 & 21.9 & 1.13 & 3.0 & 536.0 & 91.1 & 45.18 & 7008.0 \\
\hline & HS-CC & CR6-C-4-3 & 612.0 & 204.0 & 5.95 & 32.3 & 204.0 & 5.95 & 32.3 & 1.68 & 3.0 & 540.0 & 39.1 & 29.60 & 4026.0 \\
\hline & HS-HC & CR6-C-9 & 612.0 & 204.0 & 5.95 & 32.3 & 204.0 & 5.95 & 32.3 & 1.68 & 3.0 & 540.0 & 91.1 & 45.18 & 5303.0 \\
\hline & HS-CC & CR8-A-4-3 & 540.0 & 180.0 & 9.45 & 17.0 & 180.0 & 9.45 & 17.0 & 1.09 & 3.0 & 825.0 & 39.1 & 29.60 & 6803.0 \\
\hline & HS-HC & CR8-A-9 & 540.0 & 180.0 & 9.45 & 17.0 & 180.0 & 9.45 & 17.0 & 1.09 & 3.0 & 825.0 & 91.1 & 45.18 & 7402.0 \\
\hline & HS-CC & CR8-C-4-3 & 540.0 & 180.0 & 6.60 & 25.3 & 180.0 & 6.60 & 25.3 & 1.62 & 3.0 & 824.0 & 39.1 & 29.60 & 5028.0 \\
\hline & HS-HC & CR8-C-9 & 540.0 & 180.0 & 6.60 & 25.3 & 180.0 & 6.60 & 25.3 & 1.62 & 3.0 & 824.0 & 91.1 & 45.18 & 5873.0 \\
\hline \multirow{10}{*}{ Liu (2005) } & CS-HC & R7-1 & 320.0 & 106.0 & 4.00 & 24.5 & 106.0 & 4.00 & 24.5 & 1.22 & 3.0 & 495.0 & 89.0 & 44.65 & 1749.0 \\
\hline & CS-HC & R7-2 & 320.0 & 106.0 & 4.00 & 24.5 & 106.0 & 4.00 & 24.5 & 1.22 & 3.0 & 495.0 & 89.0 & 44.65 & 1824.0 \\
\hline & CS-HC & R8-1 & 390.0 & 130.0 & 4.00 & 30.5 & 90.0 & 4.00 & 20.5 & 1.52 & 4.3 & 495.0 & 89.0 & 44.65 & 1752.0 \\
\hline & CS-HC & R8-2 & 390.0 & 130.0 & 4.00 & 30.5 & 90.0 & 4.00 & 20.5 & 1.52 & 4.3 & 495.0 & 89.0 & 44.65 & 1806.0 \\
\hline & CS-HC & R9-1 & 480.0 & 160.0 & 4.00 & 38.0 & 80.0 & 4.00 & 18.0 & 1.89 & 6.0 & 495.0 & 89.0 & 44.65 & 1878.0 \\
\hline & CS-HC & R9-1 & 480.0 & 160.0 & 4.00 & 38.0 & 80.0 & 4.00 & 18.0 & 1.89 & 6.0 & 495.0 & 89.0 & 44.65 & 1858.0 \\
\hline & CS-HC & R10-1 & 420.0 & 140.0 & 4.00 & 33.0 & 140.0 & 4.00 & 33.0 & 1.64 & 3.0 & 495.0 & 89.0 & 44.65 & 2752.0 \\
\hline & CS-HC & R10-2 & 420.0 & 140.0 & 4.00 & 33.0 & 140.0 & 4.00 & 33.0 & 1.64 & 3.0 & 495.0 & 89.0 & 44.65 & 2828.0 \\
\hline & CS-HC & R11-1 & 480.0 & 160.0 & 4.00 & 38.0 & 125.0 & 4.00 & 29.3 & 1.89 & 3.8 & 495.0 & 89.0 & 44.65 & 2580.0 \\
\hline & $\mathrm{CS}-\mathrm{HC}$ & R11-1 & 480.0 & 160.0 & 4.00 & 38.0 & 125.0 & 4.00 & 29.3 & 1.89 & 3.8 & 495.0 & 89.0 & 44.65 & 2674.0 \\
\hline \multirow{8}{*}{$\begin{array}{l}\text { Aslani et } \\
\text { al. (2015) }\end{array}$} & HS-CC & HSSC 1 & 330.0 & 110.0 & 5.00 & 20.0 & 110.0 & 5.00 & 20.0 & 1.18 & 3.0 & 701.0 & 54.5 & 34.94 & 2203 \\
\hline & HS-CC & HSSC2 & 330.0 & 110.0 & 5.00 & 20.0 & 110.0 & 5.00 & 20.0 & 1.18 & 3.0 & 701.0 & 54.5 & 34.94 & 2234.0 \\
\hline & HS-CC & HSSC3 & 420.0 & 140.0 & 5.00 & 26.0 & 140.0 & 5.00 & 26.0 & 1.54 & 3.0 & 701.0 & 54.5 & 34.94 & 2942.0 \\
\hline & HS-CC & HSSC4 & 420.0 & 140.0 & 5.00 & 26.0 & 140.0 & 5.00 & 26.0 & 1.54 & 3.0 & 701.0 & 54.5 & 34.94 & 2840.0 \\
\hline & HS-CC & HSSC5 & 510.0 & 170.0 & 5.00 & 32.0 & 170.0 & 5.00 & 32.0 & 1.89 & 3.0 & 701.0 & 54.5 & 34.94 & 3118.0 \\
\hline & HS-CC & HSSC6 & 510.0 & 170.0 & 5.00 & 32.0 & 170.0 & 5.00 & 32.0 & 1.89 & 3.0 & 701.0 & 54.5 & 34.94 & 3243.0 \\
\hline & HS-CC & HSSC7 & 570.0 & 190.0 & 5.00 & 36.0 & 190.0 & 5.00 & 36.0 & 2.13 & 3.0 & 701.0 & 54.5 & 34.94 & 3882.0 \\
\hline & HS-CC & HSSC 8 & 570.0 & 190.0 & 5.00 & 36.0 & 190.0 & 5.00 & 36.0 & 2.13 & 3.0 & 701.0 & 54.5 & 34.94 & 3856.0 \\
\hline \multirow{15}{*}{$\begin{array}{l}\text { Xiong et } \\
\text { al. (2017) }\end{array}$} & HS-HC & S1 & 450.0 & 150.0 & 8.00 & 16.8 & 150.0 & 8.00 & 16.8 & 1.05 & 3.0 & 779.0 & 152.3 & 58.41 & 6536.0 \\
\hline & HS-HC & S2 & 450.0 & 150.0 & 8.00 & 16.8 & 150.0 & 8.00 & 16.8 & 1.05 & 3.0 & 779.0 & 157.2 & 59.34 & 6715.0 \\
\hline & HS-HC & S3 & 450.0 & 150.0 & 8.00 & 16.8 & 150.0 & 8.00 & 16.8 & 1.05 & 3.0 & 779.0 & 147.0 & 57.39 & 6616.0 \\
\hline & HS-HC & $\mathrm{S} 4$ & 450.0 & 150.0 & 8.00 & 16.8 & 150.0 & 8.00 & 16.8 & 1.05 & 3.0 & 779.0 & 164.1 & 60.63 & 7276.0 \\
\hline & HS-HC & S5 & 450.0 & 150.0 & 8.00 & 16.8 & 150.0 & 8.00 & 16.8 & 1.05 & 3.0 & 779.0 & 148.0 & 57.58 & 6974.0 \\
\hline & HS-HC & S6 & 450.0 & 150.0 & 12.00 & 10.5 & 150.0 & 12.00 & 10.5 & 0.65 & 3.0 & 756.0 & 152.3 & 58.41 & 8585.0 \\
\hline & HS-HC & S7 & 450.0 & 150.0 & 12.00 & 10.5 & 150.0 & 12.00 & 10.5 & 0.65 & 3.0 & 756.0 & 157.2 & 59.34 & 8452.0 \\
\hline & HS-HC & S8 & 450.0 & 150.0 & 12.00 & 10.5 & 150.0 & 12.00 & 10.5 & 0.65 & 3.0 & 756.0 & 147.0 & 57.39 & 8687.0 \\
\hline & HS-HC & S9 & 450.0 & 150.0 & 12.00 & 10.5 & 150.0 & 12.00 & 10.5 & 0.65 & 3.0 & 756.0 & 164.1 & 60.63 & 8730.0 \\
\hline & HS-HC & S10 & 450.0 & 150.0 & 12.00 & 10.5 & 150.0 & 12.00 & 10.5 & 0.65 & 3.0 & 756.0 & 148.0 & 57.58 & 8912.0 \\
\hline & CS-HC & S11 & 450.0 & 150.0 & 12.50 & 10.0 & 150.0 & 12.50 & 10.0 & 0.47 & 3.0 & 446.0 & 152.3 & 58.41 & 5953.0 \\
\hline & CS-HC & S12 & 450.0 & 150.0 & 12.50 & 10.0 & 150.0 & 12.50 & 10.0 & 0.47 & 3.0 & 446.0 & 157.2 & 59.34 & 5911.0 \\
\hline & CS-HC & S13 & 450.0 & 150.0 & 12.50 & 10.0 & 150.0 & 12.50 & 10.0 & 0.47 & 3.0 & 446.0 & 147.0 & 57.39 & 6039.0 \\
\hline & CS-HC & S14 & 450.0 & 150.0 & 12.50 & 10.0 & 150.0 & 12.50 & 10.0 & 0.47 & 3.0 & 446.0 & 164.1 & 60.63 & 6409.0 \\
\hline & $\mathrm{CS}-\mathrm{HC}$ & $\mathrm{S} 15$ & 450.0 & 150.0 & 12.50 & 10.0 & 150.0 & 12.50 & 10.0 & 0.47 & 3.0 & 446.0 & 148.0 & 57.58 & 6285.0 \\
\hline
\end{tabular}

rectangular CFT members, for example: (i) an idealized bilinear curve was used to specify the steel uniaxial stress-strain behavior in both compression and tension, and (ii) residual stresses were not included because they influence the behavior of intermediate and long columns, but have negligible influence on short columns. The only exception is the concrete material model, which is explained as follows.

In the previous research [1] conducted by the authors, the concrete damaged plasticity (CDP) material model developed by Lee and Fenves [25] was used to model the material multiaxial behavior of conventional-strength concrete. This model requires the following input: (i) multiaxial plasticity parameters including the dilation angle $(\psi)$, eccentricity $(\epsilon)$, concrete biaxial-to-uniaxial compressive strength ratio $f^{\prime}{ }_{b c} / f^{\prime}{ }_{c}$, and the ratio of compressive to tensile meridians of the yield surface in $\Pi$ (deviatoric stress) space $K_{c}$; (ii) uniaxial compressive behavior; and (iii) uniaxial tensile behavior. These input were selected as follows to model conventional-strength concrete: (i) $\psi=15^{\circ}$ and default values in ABAQUS for $\epsilon, f^{\prime}{ }_{b c} / f^{\prime}$, and $K_{c}$ (i.e., $\epsilon=0.1$, $f^{\prime}{ }_{b c} / f^{\prime}{ }_{c}=1.16$, and $K_{c}=0.67$ ); (ii) the empirical model proposed by Popovics [26] to define the uniaxial compressive behavior; and (iii) the empirical models developed by CEB-FIP [27] to define the smeared cracking behavior in tension. 
Table 2. High-strength rectangular CFT column tests: slender columns $(L / H>6.0)$.

\begin{tabular}{|c|c|c|c|c|c|c|c|c|c|c|c|c|c|c|c|c|}
\hline Reference & $\begin{array}{c}\text { Specimen } \\
\text { Type }\end{array}$ & Specimen ID & $\begin{array}{c}L \\
(\mathrm{~mm})\end{array}$ & $\begin{array}{c}B \\
(\mathrm{~mm})\end{array}$ & $\begin{array}{c}t_{f} \\
(\mathrm{~mm})\end{array}$ & $b / t_{f}$ & $\begin{array}{c}H \\
(\mathrm{~mm})\end{array}$ & $\begin{array}{c}t_{w} \\
(\mathrm{~mm})\end{array}$ & $h / t_{w}$ & $\lambda_{\text {coeff }}$ & $L / H$ & $\begin{array}{c}F_{y} \\
(\mathrm{MPa})\end{array}$ & $\begin{array}{c}f_{c}^{\prime} \\
(\mathrm{MPa})\end{array}$ & $\begin{array}{c}E_{c} \\
(\mathrm{GPa})\end{array}$ & $\begin{array}{c}P_{n} \\
(\mathrm{kN})\end{array}$ & $\begin{array}{l}P_{\text {exp }} \\
(\mathrm{kN})\end{array}$ \\
\hline Cederwall & $\mathrm{CS}-\mathrm{HC}$ & $14-2$ & 3000.0 & 120.0 & 8.00 & 13.0 & 120.0 & 8.00 & 13.0 & 0.57 & 25.0 & 379.0 & 80.0 & 42.33 & 1368.0 & 1610.0 \\
\hline \multirow{2}{*}{$\begin{array}{c}\text { Liu et al. } \\
2003\end{array}$} & HS-HC & C11-2 & 600.0 & 200.2 & 4.18 & 45.9 & 98.9 & 4.18 & 21.7 & 2.41 & 6.1 & 550.0 & 70.8 & 39.83 & 2313.3 & 2380.0 \\
\hline & $\mathrm{HS}-\mathrm{HC}$ & $\mathrm{C} 12-2$ & 600.0 & 199.8 & 4.18 & 45.8 & 99.6 & 4.18 & 21.8 & 2.40 & 6.0 & 550.0 & 82.1 & 42.89 & 2481.0 & 2800.0 \\
\hline \multirow{12}{*}{$\begin{array}{l}\text { Lue et al. } \\
2007\end{array}$} & $\mathrm{CS}-\mathrm{HC}$ & $\mathrm{C} 10^{\mathrm{K}} 6-1-6-1$ & 1855.0 & 150.0 & 4.50 & 31.3 & 100.0 & 4.50 & 20.2 & 1.37 & 18.6 & 379.8 & 70.0 & 31.94 & 1252.8 & 1894.6 \\
\hline & CS-HC & $\mathrm{C} 10^{\mathrm{K}} 6-1-6-2$ & 1855.0 & 150.0 & 4.50 & 31.3 & 100.0 & 4.50 & 20.2 & 1.37 & 18.6 & 379.8 & 70.0 & 31.94 & 1252.8 & 1889.2 \\
\hline & CS-HC & $\mathrm{C} 10^{\mathrm{K}} 6-1-6-3$ & 1855.0 & 150.0 & 4.50 & 31.3 & 100.0 & 4.50 & 20.2 & 1.37 & 18.6 & 379.8 & 70.0 & 31.94 & 1252.8 & 1885.6 \\
\hline & CS-HC & $\mathrm{C} 10^{\mathrm{K}} 6-1-6-4$ & 1855.0 & 150.0 & 4.50 & 31.3 & 100.0 & 4.50 & 20.2 & 1.37 & 18.6 & 379.8 & 70.0 & 31.94 & 1252.8 & 1891.6 \\
\hline & CS-HC & $\mathrm{C} 10^{\mathrm{K}} 6-1-6-5$ & 1855.0 & 150.0 & 4.50 & 31.3 & 100.0 & 4.50 & 20.2 & 1.37 & 18.6 & 379.8 & 70.0 & 31.94 & 1252.8 & 1862.3 \\
\hline & CS-HC & $\mathrm{C} 10^{\mathrm{K}} 6-1-6-6$ & 1855.0 & 150.0 & 4.50 & 31.3 & 100.0 & 4.50 & 20.2 & 1.37 & 18.6 & 379.8 & 70.0 & 31.94 & 1252.8 & 1889.8 \\
\hline & CS-HC & $\mathrm{C} 12^{\mathrm{K}} 6-1-6-1$ & 1855.0 & 150.0 & 4.50 & 31.3 & 100.0 & 4.50 & 20.2 & 1.37 & 18.6 & 379.8 & 84.0 & 32.84 & 1345.0 & 2066.1 \\
\hline & CS-HC & $\mathrm{C} 12^{\mathrm{K}} 6-1-6-2$ & 1855.0 & 150.0 & 4.50 & 31.3 & 100.0 & 4.50 & 20.2 & 1.37 & 18.6 & 379.8 & 84.0 & 32.84 & 1345.0 & 2196.4 \\
\hline & CS-HC & $\mathrm{C} 12^{\mathrm{K}} 6-1-6-3$ & 1855.0 & 150.0 & 4.50 & 31.3 & 100.0 & 4.50 & 20.2 & 1.37 & 18.6 & 379.8 & 84.0 & 32.84 & 1345.0 & 2096.1 \\
\hline & $\mathrm{CS}-\mathrm{HC}$ & $\mathrm{C} 12^{\mathrm{K}} 6-1-6-4$ & 1855.0 & 150.0 & 4.50 & 31.3 & 100.0 & 4.50 & 20.2 & 1.37 & 18.6 & 379.8 & 84.0 & 32.84 & 1345.0 & 2090.1 \\
\hline & $\mathrm{CS}-\mathrm{HC}$ & $\mathrm{C} 12^{\mathrm{K}} 6-1-6-5$ & 1855.0 & 150.0 & 4.50 & 31.3 & 100.0 & 4.50 & 20.2 & 1.37 & 18.6 & 379.8 & 84.0 & 32.84 & 1345.0 & 2006.7 \\
\hline & $\mathrm{CS}-\mathrm{HC}$ & $\mathrm{C} 12^{\mathrm{K}} 6-1-6-6$ & 1855.0 & 150.0 & 4.50 & 31.3 & 100.0 & 4.50 & 20.2 & 1.37 & 18.6 & 379.8 & 84.0 & 32.84 & 1345.0 & 2083.5 \\
\hline \multirow{6}{*}{$\begin{array}{c}\text { Khan et } \\
\text { al. (2017) }\end{array}$} & HS-HC & CB30-SL1 (A) & 1514.0 & 159.6 & 4.93 & 30.4 & 159.6 & 4.93 & 30.4 & 1.88 & 9.5 & 762.0 & 113.0 & 50.31 & 4032.3 & 5164 \\
\hline & HS-HC & CB30-SL1 (B) & 1514.0 & 159.3 & 4.94 & 30.2 & 159.3 & 4.94 & 30.2 & 1.87 & 9.5 & 762.0 & 113.0 & 50.31 & 4019.2 & 4833 \\
\hline & HS-HC & CB30-SL1 (C) & 1514.0 & 159.5 & 4.95 & 30.2 & 159.5 & 4.95 & 30.2 & 1.87 & 9.5 & 762.0 & 113.0 & 50.31 & 4033.1 & 5085 \\
\hline & HS-HC & CB40-SL1 (A) & 1514.0 & 208.7 & 4.91 & 40.5 & 208.7 & 4.91 & 40.5 & 2.50 & 7.3 & 762.0 & 113.0 & 50.31 & 6360.3 & 7478 \\
\hline & HS-HC & CB40-SL1 (B) & 1514.0 & 208.8 & 4.92 & 40.4 & 208.8 & 4.92 & 40.4 & 2.50 & 7.2 & 762.0 & 113.0 & 50.31 & 6372.3 & 7506 \\
\hline & HS-HC & CB40-SL1 (C) & 1514.0 & 208.8 & 4.94 & 40.3 & 208.8 & 4.94 & 40.3 & 2.49 & 7.2 & 762.0 & 113.0 & 50.31 & 6388.4 & 6460 \\
\hline
\end{tabular}

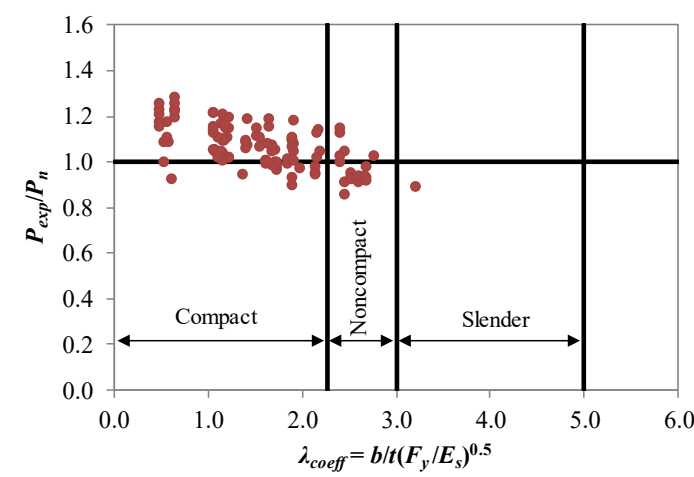

(a) Short columns $(L / H \leq 6.0)$.

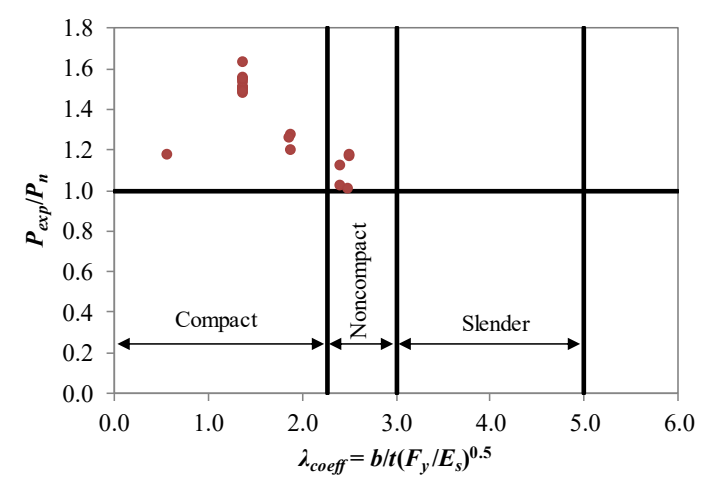

(b) Slender columns $(L / H>6.0)$.

Fig. 1. Evaluations of the AISC 360-16 design equations.

However, as explained previously by the authors [28], the CDP model in ABAQUS cannot fully account for the beneficial effects of confinement on the post-peak behavior of highstrength concrete. This results in inaccurate (more negative) predictions of the post-peak stress-strain behavior of the concrete infill for CFT columns. Researchers have proposed different solutions to address this issue. For example, Tao et al. [29] conducted series of sensitivity analyses and concluded that: (i) default values of $f^{\prime}{ }_{b c} / f^{\prime}{ }^{\prime}$ and $K_{c}$ are not suitable for modeling the concrete infill of CFT columns, and (ii) the specified concrete uniaxial compressive behavior should be modified to account for the effect of confinement. It should be noted that modifying the concrete uniaxial compressive behavior to account for confinement was more of a modeling technique rather than the original intent of the CDP model.

According to Tao et al. [29], the dilation angle $(\psi)$ for rectangular CFT columns can be set as $40^{\circ}$, the $f^{\prime}{ }^{\prime} d f^{\prime}{ }_{c}$ ratio can be determined using Eq. (1) as proposed by Papanikolaou and Kappos [30], and $K_{c}$ can be determined using Eq. (2) based on Yu et al. [31]. Lower values of $K_{c}$ result in increase of strength and less negative postpeak stiffness.

$$
\frac{f_{b c}^{\prime}}{f^{\prime}{ }_{c}}=1.5\left(f_{c}^{\prime}\right)^{-0.075}
$$




$$
K_{c}=\frac{5.5}{5+2\left(f_{c}^{\prime}\right)^{-0.075}}
$$

Tao et al. [29] also proposed a stress-strain relationship to include the effect of confinement on the uniaxial compressive behavior of the concrete infill, as shown in Fig. 2. This relationship consists of an ascending branch $\mathrm{OA}$, a plateau $\mathrm{AB}$, and a descending branch $\mathrm{BC}$. The ascending branch $\mathrm{OA}$ is defined by Eq. (3), where parameters $m$ and $n$ determine the shape, $\varepsilon_{c}$ is the strain at peak stress, and $E_{c}$ is the concrete elastic modulus determined as per ACI 318-14 [32]. The plateau branch $\mathrm{AB}$ is determined by the strain at point $\mathrm{B}\left(\varepsilon_{c c}\right)$, which is defined using Eq. (4). The descending branch $\mathrm{BC}$ is defined by Eq. (5), where parameters $\alpha_{c}$ and $\beta_{c}\left(\beta_{c}=0.92\right.$ for rectangular CFT columns) determine the shape, $f_{r}$ represents the residual (stabilized) strength at point $\mathrm{C}, A_{c}$ is the crosssection area of the concrete infill, and $A_{s}$ is the cross-section area of the steel tube. It should be noted that values of $\psi, f_{B}, f_{r}, \alpha$, and $\beta_{c}$ for circular CFT columns are different from those for rectangular CFT columns.

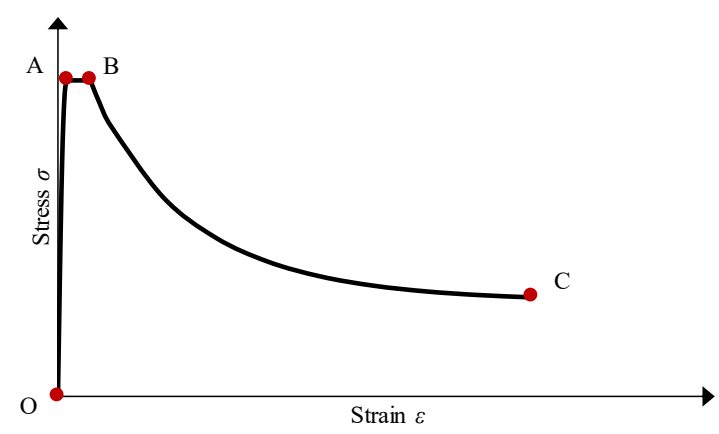

Fig. 2. Compressive stress-strain relationships for concrete

$\frac{\sigma}{f_{c}^{\prime}}=\frac{m \cdot X+n \cdot X^{2}}{1+(m-2) X+(n+1) X^{2}}$

$X=\frac{\varepsilon}{\varepsilon_{c}}$

$m=\frac{E_{c} \varepsilon_{c}}{f^{\prime}{ }_{c}}$

$n=\frac{(m-1)^{2}}{0.55}-1$

$\varepsilon_{c}=0.00076+\sqrt{\left(0.626 f_{c}^{\prime}-4.33\right) \times 10^{-7}}$
$E_{c}=4700 \sqrt{f^{\prime}{ }_{c}}$
$\varepsilon_{c c}=e^{k} \varepsilon_{c}$

$k=\left(2.9224-0.00367 f_{c}^{\prime}{ }_{c}\right)\left(\frac{f_{B}}{f^{\prime}{ }_{c}}\right)^{0.3124+0.002 f^{\prime} c}$

$f_{B}=\frac{0.25\left(1+0.027 F_{y}\right) e^{\frac{-0.02 \sqrt{B^{2}+D^{2}}}{t}}}{1+1.6 e^{-10}\left(f_{c}^{\prime}\right)^{4.8}}$

$\sigma=f_{r}+\left(f^{\prime}{ }_{c}-f_{r}\right) \exp \left\lfloor-\left(\frac{\varepsilon-\varepsilon_{c c}}{\alpha_{c}}\right)^{\beta_{c}}\right\rfloor$

$f_{r}=0.1 f_{c}^{\prime}$

$\alpha_{c}=0.005+0.0075 c_{s r}$

$c_{s r}=\frac{A_{s} F_{y}}{A_{c} f^{\prime}{ }_{c}}$

Thus, the CDP model was used for the material multiaxial behavior of concrete for high-strength rectangular CFT columns. The default value in ABAQUS was used for eccentricity $(\epsilon=0.1)$. The dilation angle $(\psi)$, concrete biaxial-to-uniaxial compressive strength ratio $f^{\prime}{ }_{b d} / f^{\prime}{ }_{c}$, and the ratio of compressive to tensile meridians of the yield surface in $\Pi$ space $\left(K_{c}\right)$ were determined based on the recommendations by Tao et al. [29], i.e., $\psi=40^{\circ}, f^{\prime}{ }_{b c} / f^{\prime}{ }_{c}$ defined by Eq. (1), and $K_{c}$ defined by Eq. (2). Also, the Tao et al. [29] stress-strain relationship was used to specify the concrete uniaxial behavior in compression. Similar to [1], the concrete tensile stress-crack opening displacement behavior was specified using the empirical model developed by CEB-FIP [27].

The developed FEM models were benchmarked by using them to predict the behavior and strengths of the 130 high-strength CFT columns in the experimental database. Fig. 3 shows the resulting comparisons. As shown, the mean experimental-to-predicted strength ratio $\left(P_{\text {exp }} / P_{F E M}\right)$ ratio is 1.05 , and the corresponding coefficient of variation is 0.085 . Fig. 4 shows representative comparisons of the axial force-displacement responses. Figs. 3 and 4 indicate that the FEM models can reasonably predict the behavior and strengths of highstrength rectangular CFT columns. 


\section{Summary and conclusions}

This paper presented the results of a systematic approach that was used to address gaps in AISC 360-16 for designing high-strength rectangular CFT columns. In the first step, an experimental database consisting of 130 highstrength rectangular CFT column tests was compiled. Test results from the database were used to evaluate the possibility of extending the AISC 360-16 design equations for estimating the strength of high-strength rectangular CFT short columns. The evaluations indicated that AISC design equations reasonably estimated the strength of high-strength rectangular CFT short columns with compact sections. However, they were slightly unconservative for those with noncompact sections.

In the second step, detailed 3D nonlinear FEM models for high-strength CFT columns were developed and benchmarked. The benchmarked models are currently being used to perform parametric studies to (i) address gaps in the database and (ii) propose design equations for high-strength rectangular CFT members, which will be part of a future paper.

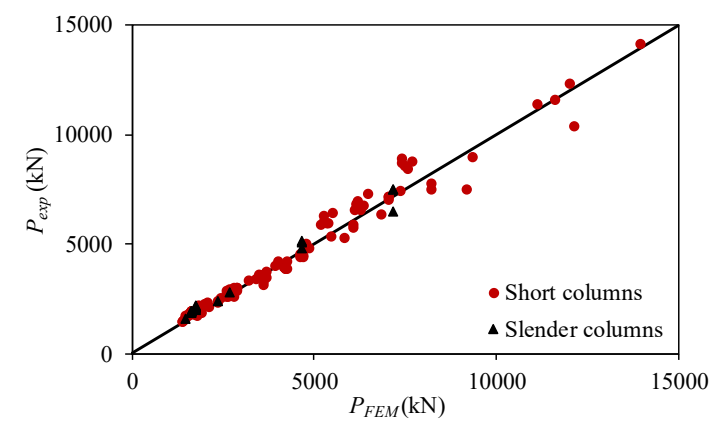

Fig. 3. Comparisons of the strengths obtained from the finite element analyses and corresponding tests.

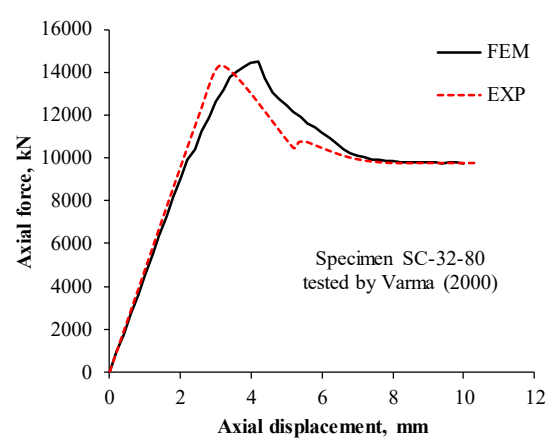

(a)

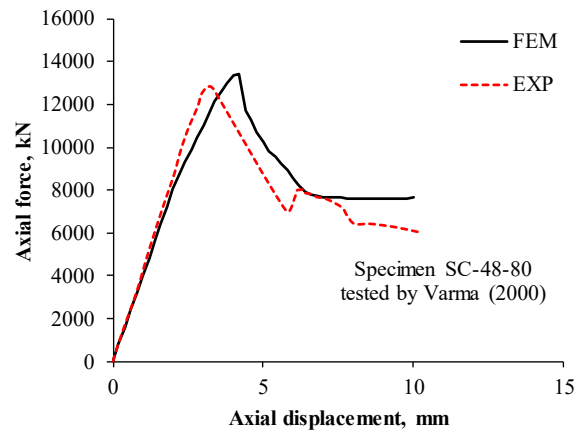

(b)

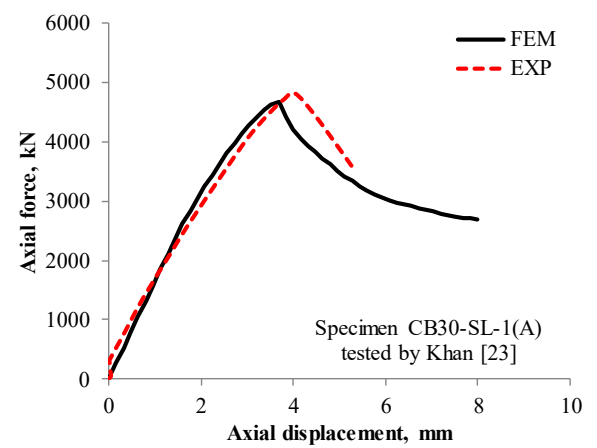

(c)

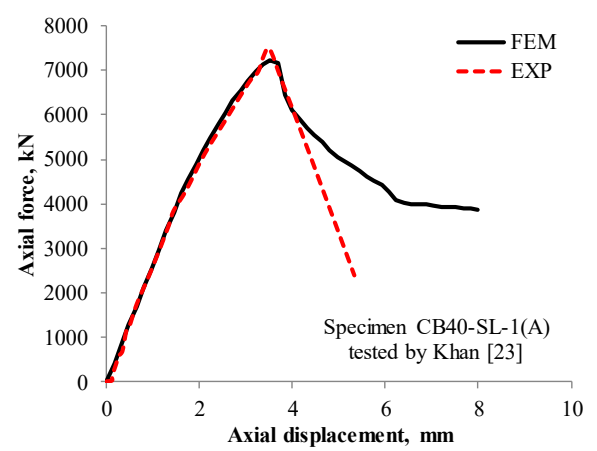

(d)

Fig. 4. Representative comparisons of the axial force-displacement responses: (a) and (b) short columns, (c) and (d) slender columns.

\section{References}

[1] Lai Z, Varma AH, Zhang K. Noncompact and slender rectangular CFT members: experimental database, analysis, and design. Journal of Constructional Steel Research 2014;101:455-68.

[2] Lai Z, Varma AH. Noncompact and slender circular CFT members: Experimental database, analysis, and design. Journal of Constructional Steel Research 2015; 106: 220-33.

[3] Lai Z, Varma AH, Griffis LG. Analysis and design of noncompact and slender CFT beamcolumns. Journal of Structural Engineering 2016; 142.

[4] Huang W, Lai Z, Chen B, Xie Z, Varma AH. Concrete-filled steel tube (CFT) truss girders: experimental tests, analysis, and design. Engineering Structures 2017; 156: 118-29. 
[5] Lai Z, Huang Z, Varma AH. Seismic analysis and performance of high strength composite special moment frames (C-SMFs). Structures 2016; 9: 165-78.

[6] Lai Z, Varma AH. Seismic behavior and modeling of circular concrete partially filled spirally welded pipes (CPFSWP). Thin-Walled Structures 2017; 113: 240-52.

[7] Chen B, Lai Z, Yan Q, Varma AH, Yu X. Experimental behavior and design of CFT-RC short columns subjected to concentric axial loading. Journal of Structural Engineering 2017; 143.

[8] AISC 360-16. Specification for structural steel buildings. Chicago, IL, USA: AISC; 2016.

[9] Nishiyama I, Morino S, Sakino K, Nakahara H, Fujimoto T, Mukai A, et al. Summary of research on concrete-filled structural steel tube column system carried out under the U.S.-Japan cooperative research on composite and hybrid structures. Ibaraki Prefecture, Japan: 2002.

[10] Kim DK. A database for composite columns [Thesis]. Georgia Institute of Technology; 2005.

[11]Hajjar JF, Gourley BC, Tort C, Denavit MD, Schiller PH. Steel-concrete composite structural systems,

http://www.northeastern.edu/compositesystems. Northeastern University; 2013.

[12] Lai Z, Varma AH. Noncompact and slender circular CFT members: experimental database, analysis, and design. Journal of Constructional Steel Research 2015; 106: 220-33.

[13] Cederwall K, Engstrom B, Grauers M. Highstrength concrete used in composite columns. In: Hester TT, editor. Proceedings of the 2nd International Symposium on utilization of High Strength Concrete, Berkeley, CA, USA: 1990, 195-214.

[14]Varma AH. Seismic behavior, analysis and design of high strength square concrete filled steel tube (CFT) columns [Dissertation]. Lehigh University, 2000.

[15] Uy B. Strength of short concrete filled high strength steel box columns. Journal of Constructional Steel Research 2001; 57: 113-34.

[16] Liu D, Gho WM, Yuan J. Ultimate capacity of high-strength rectangular concrete-filled steel hollow section stub columns. Journal of Constructional Steel Research 2003; 59: 1499 515.

[17] Mursi M, Uy B. Strength of slender concrete filled high strength steel box columns. Journal of Constructional Steel Research 2004;60:1825-48.

[18] Sakino K, Nakahara H, Morino S, Nishiyama A. Behavior of centrally loaded concrete-filled steel-tube short columns. Journal of Structural
Engineering 2004; 130: 180-188.

[19]Liu D. Tests on high-strength rectangular concrete-filled steel hollow section stub columns. Journal of Constructional Steel Research 2005; 61: 902-11.

[20]Lue DM, Liu J-L, Yen T. Experimental study on rectangular CFT columns with high-strength concrete. Journal of Constructional Steel Research 2007; 63: 37-44.

[21] Aslani F, Uy B, Tao Z, Mashiri F. Behaviour and design of composite columns incorporating compact high-strength steel plates. Journal of Constructional Steel Research 2015;107:94-110.

[22]Xiong M-X, Xiong D-X, Liew JYR. Axial performance of short concrete filled steel tubes with high- and ultra-high- strength materials. Engineering Structures 2017; 136: 494-510.

[23] Khan M, Uy B, Tao Z, Mashiri F. Behaviour and design of short high-strength steel welded box and concrete-filled tube (CFT) sections. Engineering Structures 2017; 147: 458-72.

[24] ABAQUS. ABAQUS Version 6.16 Analysis User's Manuals. Providence, RI, USA: Dassault Systemes Simulia Corporation; 2016.

[25]Lee J, Fenves GL. Plastic-damage model for cyclic loading of concrete structures. Journal of Engineering Mechanics 1998; 124: 892-900.

[26] Popovics S. A numerical approach to the complete stress-strain curve of concrete. Cement and Concrete Research 1973; 3: 583-99.

[27] CEB-FIP (Euro-International Committee for Concrete (CEB)-International Federation for Prestressing (FIP).). Model Code for concrete structures. (CEB-FIP MC 2010). London, U.K.: Thomas Telford; 2010.

[28]Lai Z, Varma AH. Effective stress-strain relationships for noncompact and slender CFT members. Engineering Structures 2016; 124: 457-72.

[29] Tao Z, Wang Z-B, Yu Q. Finite element modeling of concrete-filled steel stub columns under axial compression. Journal of Constructional Steel Research 2013;89:121-31.

[30] Papanikolaou VK, Kappos AJ. Confinementsensitive plasticity constitutive model for concrete in triaxial compression. International Journal of Solids and Structures 2007; 44: 702148.

[31] Yu T, Teng JG, Wong YL, Dong SL. Finite element modeling of confined concrete-I: Drucker-Prager type plasticity model. Engineering Structures 2010; 32: 665-79.

[32] ACI 318-14. Building code requirements for structural concrete. Farmington Hills, MI, USA: ACI; 2014. 